\title{
Spatial frequency masking of lateralized word recognition
}

\author{
DAVID B. BOLES and RAHEEL RASHID \\ Rensselaer Polytechnic Institute, Troy, New York
}

\begin{abstract}
Lateralized words varying in size were postexposurally masked using checkerboards of 2,4 , or 8 cycles per degree. In errors, main effects were found for size, visual field, and spatial frequency. Although the expected right visual field advantage in recognition was found, and the differential effectiveness of the spatial-frequency masks was supported, there was no visual field $x$ frequency interaction. In reaction time, a size $\times$ visual field interaction was found, but it was opposite to the effect typically claimed for size as an input variable. It is concluded that views of hemispheric differences that stress process type are likely to have more explanatory value than those stressing spatial frequency.
\end{abstract}

One hypothesis of hemispheric differences in processing visual information is that the two hemispheres differ in sensitivity to spatial frequency. The left hemisphere, it is supposed, is more sensitive to high frequencies, so that when stimuli are presented to one visual field or the other and require processing of high frequencies for recognition, a right visual field (RVF) advantage typically results. Conversely, stimuli requiring processing of low frequencies are said to typically result in left visual field (LVF) advantages (Sergent, 1982).

Although some research supports this spatial-frequency distinction between hemispheres (Kitterle, Hellige, \& Christman, 1992; Sergent, 1985), other studies fail to do so (Fendrich \& Gazzaniga, 1990; Hardyck, 1991; Peterzell, Harvey, \& Hardyck, 1989). Boles and Morelli (1988) used square-wave gratings in tasks requiring the samedifferent matching of spatial frequency and the enumeration of bars within gratings. Although the same-different task produced a visual field by spatial-frequency interaction, it was not of the type predicted, since the lowest frequencies $(0.5,0.6$, and 0.8 cycles per degree; cpd) showed no visual field asymmetry, whereas some of the middle and high frequencies $(1.3,2.5,10.1$, and $20.0 \mathrm{cpd})$ showed LVF advantages. In the enumeration task, a generalized LVF advantage was seen regardless of frequency (ranging from 0.5 to $5.0 \mathrm{cpd}$ ). These results were viewed as problematic for the spatial-frequency hypothesis.

One shortcoming of the study by Boles and Morelli (1988) was that there was no check built into the experimental design to demonstrate that spatial frequency had been effectively manipulated. Since Fourier analysis of a square-wave grating shows that it consists of the fundamental frequency together with higher frequency harmonics (De Valois \& De Valois, 1990), it could be argued that the stimuli were not sufficiently pure to produce the

Correspondence should be addressed to David B. Boles, Department of Psychology, Rensselaer Polytechnic Institute, Troy, NY 12180 (e-mail: userbwwl@rpitsmts.bitnet). expected effects. Although it is difficult with such an argument to explain why lowering the frequency spectrum did not produce increasing LVF advantages, a check would be desirable to demonstrate that the spatial-frequency manipulation was effective.

In the present study, such a check is provided by assessing the effect of a postexposure mask on the recognition of lateralized stimuli. The spatial frequency, but not the space-averaged contrast, of the square-wave mask varies. If the masks are so impure that their frequency content fails to affect the recognition task, the effect of one mask should be equivalent to any other. On the other hand, if one frequency produces a masking effect larger than those for other frequencies, the implication would be that the manipulation was effective and that spatial frequency was relevant to performance of the recognition task. In such a case, the spatial-frequency hypothesis predicts a visual field $\times$ frequency interaction, because the masking of particular frequencies should reduce their availability for processing, shifting processing toward one or the other hemisphere accordingly.

Stimulus size was also manipulated in the study because of claims that "input variables" affect the availability of spatial frequencies (Christman, 1989; Sergent \& Hellige, 1986). Size is an input variable whose relationship to spatial frequency is particularly straightforward because large stimuli have lower frequency spectra than do small stimuli. Thus relative to small stimuli, large stimuli should result in a shift of processing toward the right hemisphere, producing a visual field $\times$ size interaction.

\section{METHOD}

\section{Subjects}

Eighteen subjects participated, 13 males and 5 females, in return for class credit. All were self-reported right-handers.

\section{Apparatus}

Stimuli were presented and responses collected using an Apple II microcomputer, a black-and-white Apple III monitor, and an external keyboard. Millisecond timing accuracy was achieved using the Apple- 
Psych system (Barnes \& Burke, 1988; Osgood, 1988). The black-andwhite monitor (P4 phosphor) had two components, blue and yellow, with respective decay times of 0.15 and $0.475 \mathrm{msec}$ (to $1 \%$ of brightness).

\section{Stimuli}

Stimuli for recognition were the word names of the numbers 1 through 8 (e.g., ONE). The words were presented in capital letters in vertical orientation at a viewing distance of $142 \mathrm{~cm}$ (enforced by chinrest). Two word sizes were used with corresponding variations of letter size and spacing. Small words subtended $0.2^{\circ}$ horizontally and between $0.7^{\circ}$ and $1.3^{\circ}$ vertically, depending on word length, whereas the corresponding figures were twice as large for the large words. Eccentricity to the near edge was $1.2^{\circ}$. Bilateral displays were used because they have been found to result in larger, more significant, and more reliable field asymmetries than have unilateral displays (Boles, 1983, 1987, 1990, in press). A bilateral display consisted of two randomly selected words in opposite visual fields, with a central arrowhead pointing to the word to be recognized.

Masks were checkerboard patterns of constant space-averaged contrast, with the pattern cycling every $1 / 2^{\circ}, 1 / 4^{\circ}$, or $1 / 8^{\circ}$ both horizontally and vertically (i.e., 2,4 or $8 \mathrm{cpd}$ ). The masks subtended $5.9^{\circ} \times$ $3.4^{\circ}$ horizontally and vertically and always overlapped the areas in which words were presented with a border to spare. However, the exact vertical and horizontal positioning of the masks, relative to the fixation and stimulus displays, was randomly perturbed on a trial-wise basis to vary the registration of edges in the masks with the stimulus words. On average across trials, the masks were nevertheless laterally symmetrical with respect to the fixation point.

\section{Procedure}

A trial began with a central fixation cross presented for $1,000 \mathrm{msec}$, followed by a 100 -msec blank, a 100 -msec bilateral word display, a 17 -msec blank, and a 100 -msec mask. The subject then responded within a deadline of $3,750 \mathrm{msec}$, using two keys in a near-far arrangement and fingers from separate hands on the near (even) and far (odd) keys. The task was to determine whether the word pointed to by the central arrowhead represented an odd (e.g., FIVE) or even (e.g., TWO) number. Reaction time (RT) feedback or the word "ERROR" was given on a trial-wise basis. Hand assignments were balanced across subjects.

The three spatial frequencies of the mask $(2,4$, and $8 \mathrm{cpd})$ were manipulated within subjects by running all trials within a condition together but balancing the condition order across subjects. Within each spatialfrequency condition, there was one block of 48 practice trials followed by two blocks of 96 experimental trials each. Thus across the 1-h session, the subjects responded to a total of 720 trials.

\section{RESULTS}

Percent errors and median RTs were calculated for each condition in the size (small vs. large words) $\times$ visual field (left vs. right) $\times$ spatial frequency $(2,4$, or 8 cpd mask) design. Separate analyses of variance were then calculated for each dependent measure. Because most effects were found in the percent errors analysis, it is presented first.

\section{Percent Errors}

All three main effects were significant. The size effect $[F(1,17)=37.21, p<.001]$ involved fewer errors for large words $(16.9 \%)$ than for small words $(21.8 \%)$. The visual field effect $[F(1,17)=5.80, p<.05]$ revealed fewer errors for right visual field $(17.9 \%)$ than for left visual field trials $(20.8 \%)$. Finally, the spatial-frequency effect $[F(2,34)=3.47, p<.05]$ consisted of fewer errors for the 4- and 8-cpd masks (18.3\% and $17.3 \%)$ than for the 2-cpd mask (22.5\%).

In spite of the established effectiveness of the three experimental manipulations, however, there were no significant interactions. Particularly notable are the low $F$ values (all $F_{\mathrm{S}}<1$ ) for the visual field $\times$ spatial frequency, size $\times$ visual field, and size $\times$ visual field $\times$ spatial-frequency terms.

\section{RTs}

Only one effect was significant, the size $\times$ visual field interaction $[F(1,17)=8.12, p=.01]$. The right visual field (RVF) advantage was more pronounced for the large words $(\mathrm{LVF}=1,109 \mathrm{msec}, \mathrm{RVF}=1,076 \mathrm{msec}$, difference $=+33 \mathrm{msec}$ ) than for the small words (LVF = $1,112 \mathrm{msec}, \mathrm{RVF}=1,104 \mathrm{msec}$, difference $=+8 \mathrm{msec}$ ). No term involving the spatial frequency variable approached significance (all $F_{\mathrm{s}}<1$ ).

\section{DISCUSSION}

Varying the spatial frequency of the checkerboard mask proved to be an effective manipulation, as indicated by the increased errors in the 2 -cpd condition. Nevertheless, there was no indication that this manipulation interacted with visual field of presentation or, by implication, with hemispheric asymmetry. According to those investigators who hypothesize a differential hemispheric sensitivity to spatial frequency, the right hemisphere is more sensitive to low spatial frequencies and the left hemisphere is more sensitive to high spatial frequencies (Kitterle et al., 1992; Sergent, 1982, 1985). Thus, to the extent that masking reduces performance, masking the low-frequency components of the word display should have resulted in impaired LVF performance relative to the RVF. No evidence for such an effect was found; instead, both VFs were equally impaired by the 2-cpd mask.

The fact that performance was impaired is important because it indicates that the 2-cd frequency was actually used in the word recognition task. In other words, it cannot be argued that the masking effect was confined to unused frequencies that did not impact word recognition and for that reason failed to result in a visual field $\times$ frequency interaction (Kitterle et al., 1992).

Manipulation of word size was likewise effective since fewer errors were found for larger words. In RT, size interacted with visual field, but not in the manner suggested by those researchers who conclude that there are input variable contributions to visual field asymmetry (Christman, 1989; Sergent \& Hellige, 1986). That is, the "expected"' effect is of a decreased RVF advantage for larger words. However, the opposite was found. This effect is likewise inconsistent with the spatialfrequency view of hemispheric differences because larger patterns have a lower spatial frequency content (thereby supposedly favoring the right hemisphere) than do smaller patterns.

Interestingly, this "reversed" input variable effect of size was also seen by Hardyck (1991) and in recent experiments (Boles, in press) comparing the contributions to visual field asymmetry of input variables (eccentricity, duration, luminance, and size), stimulus type (bar graphs vs. words), and display type (unilateral vs. bilateral displays). The conclusion from those experiments was that stimulus type determined the direction of visual field asymmetry and display type determined its magnitude, whereas input variables had little consistent effect. It is not known why the "reversed" effect of size is sometimes found, but it is not congruent with claims made in the literature.

In conclusion, it appears that asymmetries in word recognition are insensitive to the masking of particular ranges of spatial frequencies. Together with the observed failure (Boles, in press) of most input vari- 
ables to affect asymmetry for both words and bar graphs (a type of stimulus processed predominantly by the right hemisphere), a serious question is raised about the explanatory and predictive power of the spatialfrequency view of visual field asymmetries. An alternative view, that such asymmetries typically reflect types of hemispheric processes (Boles, 1991, 1992), seems considerably more powerful in this regard.

\section{REFERENCES}

Barnes, S., \& Burke, R. S. (1988). What is Apple-Psych? Behavior Research Methods, Instruments, \& Computers, 20, 150-154.

Boles, D. B. (1983). Hemispheric interaction in visual field asymmetry. Cortex, 19, 99-114.

Boles, D. B. (1987). Reaction time asymmetry through bilateral vs unilateral stimulus presentation. Brain \& Cognition, 6, 321-333.

Boles, D. B. (1990). What bilateral displays do. Brain \& Cognition, 12, 205-228.

BolEs, D. B. (1991). Factor analysis and the cerebral hemispheres: Pilot study and parietal functions. Neuropsychologia, 29, 59-91.

BoLEs, D. B. (1992). Factor analysis and the cerebral hemispheres: Temporal, occipital, and frontal functions. Neuropsychologia, 30, 963-988.

Boles, D. B. (in press). Parameters of the bilateral effect. In F. L. Kitterle (Ed.), Hemispheric transfer: Data and theory. Hillsdale, NJ: Erlbaum.

Boles, D. B., \& Morelli, M. L. (1988). Hemispheric sensitivity to spatial frequencies. Bulletin of the Psychonomic Society, 26, 552-555.

Christman, S. (1989). Perceptual characteristics in visual laterality research. Brain \& Cognition, 11, 238-257.
De Valois, R. L., \& De Valois, K. K. (1990). Spatial vision. New York: Oxford University Press

Fendrich, R., \& GAzZaniga, M. (1990). Hemispheric processing of spatial frequencies in two commissurotomy patients. Neuropsychologia, 28, 657-663.

HARDYCK, C. (1991). Shadow and substance: Attentional irrelevancies and perceptual constraints in the hemispheric processing of language stimuli. In F. L. Kitterle (Ed.), Cerebral laterality: Theory and research (pp. 133-153). Hillsdale, NJ: Erlbaum.

Kitterle, F. L., Hellige, J. B., \& Christman, S. (1992). Visual hemispheric asymmetries depend on which spatial frequencies are task relevant. Brain \& Cognition, 20, 308-314.

OsGoOD, G. (1988). Generalizing the Apple-Psych system. Behavior Research Methods, Instruments, \& Computers, 20, 155-157.

Peterzell, D. H., Harvey, L. O., Jr., \& Hardyck, C. D. (1989). Spatial frequencies and the cerebral hemispheres: Contrast sensitivity, visible persistence, and letter classification. Perception \& Psychophysics, 46, 443-455.

SERGENT, J. (1982). Theoretical and methodological consequences of variations in exposure duration in visual laterality studies. Perception \& Psychophysics, 31, 451-461.

SERGENT, J. (1985). Influence of task and input factors on hemispheric involvement in face processing. Journal of Experimental Psychology: Human Perception \& Performance, 11, 846-861.

Sergent, J., \& Hellige, J. B. (1986). Role of input factors in visualfield asymmetries. Brain \& Cognition, 5, 200-222.

(Manuscript received June 17, 1993.) 